\title{
Sciendo
}

Int. J. of Applied Mechanics and Engineering, 2020, vol.25, No.3, pp.103-116

DOI: 10.2478/ijame-2020-0037

\section{EFFECT OF CONVECTIVE HEAT AND MASS CONDITIONS IN MAGNETOHYDRODYNAMIC BOUNDARY LAYER FLOW WITH JOULE HEATING AND THERMAL RADIATION}

\author{
R.P. SHARMA* \\ Department of Mechanical Engineering \\ National Institute of TechnologyArunachal Pradesh \\ Yupia, Papum Pare District, Arunachal Pradesh-791112, INDIA \\ E-mail: rpsharma@nitap.ac.in \\ SEEMA TINKER \\ Department of Mathematics, JECRC University Jaipur-303905, India \\ B.J. GIREESHA and B. NAGARAJA \\ Department of Studies and Research in Mathematics \\ Kuvempu University \\ Shimoga-577 451, Karnataka, INDIA \\ E-mails: bjgireesu@gmail.com; nagaraj.kalapanahalli@gmail.com
}

\begin{abstract}
A free convection viscous MHD flow over a semi-infinite vertical sheet with convective heat and mass conditions has been considered. The effects of thermal radiation, chemical reaction and Joule heating on flow are also accounted. The governing nonlinear partial differential equations have been transformed into a set of highly non-linear coupled ordinary differential equations (ODEs) using appropriate similarity transformations. Numerical solutions of transformed equations are obtained by employing the $5^{\text {th }}$ order Runge-Kutta Fehlberg technique followed by the shooting technique. The influences of different flow parameters on the momentum, energy and mass field are discussed and shown graphically. Results reveal that temperature and concentration profiles enhance due to increasing heat and mass Biot number parameters.
\end{abstract}

Key words: convection, MHD, Joule heating, chemical reaction, thermal radiation.

\section{Introduction}

Convective condition at the boundary is used to characterize the convective energy transport condition throughout the surface and is directly related to the difference in fluid temperature with the ambient situation. Application of this type of condition can be seen in various manufacturing devices such as heat exchangers, gas turbines, atomic plants, thermal energy storage, and many others. Further, the use of surface convective heat along with concentration boundary conditions is a novel concept that is being inspected currently. Based on these applications, numerous investigations were carried out by various researchers. The mixed influence of natural convection, as well as heat and mass transfer on an unsteady magnetohydrodynamic motion of (Walters fluid Model-B) fluid in an asymmetrical channel under convective boundary, has been reported by Sivaraj and Rushi Kumar [1]. Thermo diffusion impact on combined convection motion of nanofluid was explored by Reddy et al. [2]. Hayat et al. [3] examined the influence of thermo-diffusion and diffusion thermo influences on Williamson liquid past an unsteady extending surface in the presence of thermal radiation, viscous dissipation and energy, and mass convective conditions. Uddin et al. [4] analytically examined the effect of Brownian and thermophoretic diffusions of natural convection motion of nanofluid in a Darcian permeable media in the presence of convective

* To whom correspondence should be addressed 
boundary conditions. Sheikholeslami et al. [5] have conducted the numerical investigation on magnetohydrodynamic free convection flow of $\mathrm{Al}_{2} \mathrm{O}_{3}$ - water-based nanofluid with thermal radiation. Sharma et al. [6] have carried out the numerical study of chemical reaction impact on magnetohydrodynamic rotating liquid past a vertical surface with variable thermal conductivity. Mishra et al. [7] examined the magnetohydrodynamic effect on viscoelastic liquid past an extending sheet. The effect of convective boundary conditions on the three-dimensional double-diffusive flow of non-Newtonian fluid motion has been examined by Gireesha et al. [8] Ramzan et al. [9] explored the impact of viscous dissipation and convective conditions on flow of Casson nanofluid.

Nowadays the investigation of prominent magnetohydrodynamic (MHD) fluid flow has engaged several researchers due to its numerous applications in different fields. Its importance on fluid flow was described by Hartman [10] during the study of electromagnetic pump fabrication. Such type of flow was utilized in the power generator system in which heat energy is converted into electric energy. A study on the MHD motion of Maxwell liquid has been investigated by Archana et al. [11] and has analyzed its impact on the velocity and temperature fields. Time-dependent MHD flow of electrically conducting Newtonian fluid has been analyzed by Makinde et al. [12]. Some more studies on MHD flow are reported by several researchers [13] - [16] ${ }^{13-16}$ under various physical conditions.

Many researchers have carried out the theoretical as well as experimental investigations on the effect of radiation in fluid flow in which they incorporated Cogley approximation of linear type. Its impact is of considerable interest in various non-isothermal conditions [17] - [20]. Further, the cause of Joule heating due to the applied electric field around conducting fluid. This impact generates temperature gradient and leads to enhanced energy which intake helps in the decomposition of thermally labile samples and the formation of gas bubbles. The role of Joule heating in different fluid flows was studied by several authors [21] - [24] under different physical situations. Kumar et al. [26] investigated the nonlinear radiation effect on nanofluid due to a stretching surface with a chemical reaction.

It has been observed that the energy and mass transfer examination in the over has been frequently dispensed under the influence of the boundary condition either via a given temperature or heat flux at the sheet. Only some works in this direction are completed considering heat convective condition at the sheet in place of prearranged sheet temperature or heat flux. Though, no effort has been up till now for the convective mass condition at the sheet. This investigation establishes such a condition in the study. Therefore, the present study is concentrated on heat and mass transfer of laminar magnetohydrodynamic motion of fluid along with convective boundary conditions. The governing dimensionless nonlinear ODEs are explained numerically by using the Runge-Kutta Fehlberg-Fifth order method with the help of the shooting method. Graphs of various interesting physical parameters are plotted for the momentum, heat and concentration profiles.

\section{Mathematical formulation}

Two-dimensional $\left(x^{*}, y^{*}\right)$ steady, laminar, MHD motion of an electrically conducting fluid past an extending sheet is addressed. Convective heat and mass conditions are taken into account. The magnetic field of strength $B_{0}$ has been is applied normal to the flow field (see Fig.1). The magnetic Reynolds number is chosen pretty small. As a consequence, the induced magnetic field is small in comparison to the applied magnetic field. Thus, the induced magnetic field is not considered. The influence of viscous dissipation and radiation is further considered. 


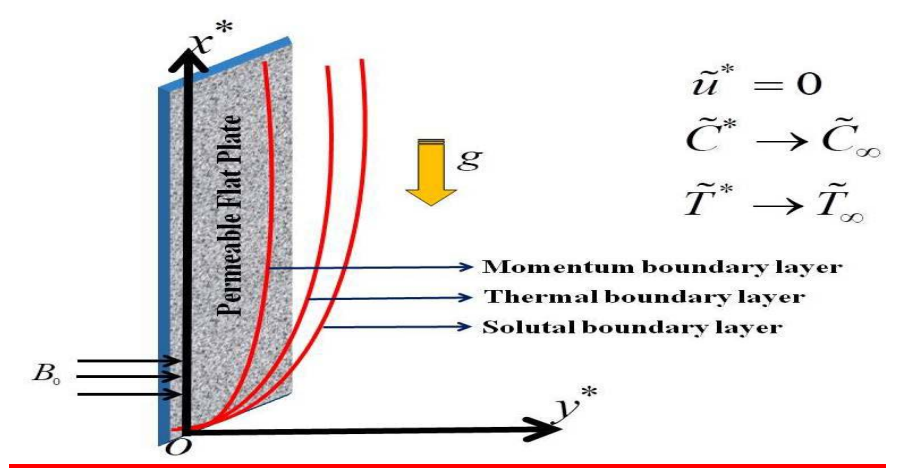

Fig.1. Flow diagram.

The two-dimensional MHD equations for formulated problem are given by

$$
\begin{aligned}
& \tilde{v}_{y}^{*}=0, \\
& \tilde{v}^{*} \tilde{u}_{\tilde{y}}^{*}=v \tilde{u}_{\tilde{y} \tilde{y}}^{*}+g \beta_{1}\left(\tilde{T}^{*}-\tilde{T}_{\infty}\right)+g \beta^{*}\left(\tilde{C}^{*}-\tilde{C}_{\infty}\right)-\frac{v}{K^{*}} \tilde{u}^{*}-\frac{\sigma B_{0}^{2} \tilde{u}^{*}}{\rho}, \\
& \tilde{v}^{*} \tilde{T}_{\tilde{y}^{*}}^{*}=\frac{\kappa}{\rho C_{p}} \tilde{T}_{\tilde{y}^{*} \tilde{y}^{*}}^{*}+\frac{v}{C_{p}}\left(\tilde{u}_{\tilde{y}}^{*}\right)^{2}-\frac{1}{\rho C_{p}} q_{\tilde{r}^{*}}-\frac{\sigma B_{0}^{2}}{\rho C_{p}} \tilde{u}^{* 2}, \\
& \tilde{v}^{*} \tilde{C}_{\tilde{y}^{*}}^{*}=D \tilde{C}_{\tilde{y}^{*} \tilde{y}^{*}}^{*}-k_{c} \tilde{C}^{*} .
\end{aligned}
$$

The solution of Eq.(2.1) is

$$
\tilde{v}^{*}=\text { cons } \tan t=-v_{0} \quad \text { and } \quad v_{0}>0 .
$$

It is emphasized by Cogley et al. [25] that the rate of radiative flux in theoptically thin limit for a non-Gray gas near equilibrium is given by

$$
q_{\tilde{y}^{*}}=4\left(\tilde{T}^{*}-\tilde{T}_{\infty}\right) I^{\prime}
$$

where $\quad I^{\prime}=\int K_{\lambda} \frac{\partial e_{\lambda}}{\partial \tilde{T}^{*}} d \lambda$.

Subjected to appropriate boundary conditions

$$
\begin{aligned}
& \tilde{u}^{*}=0, \quad k_{f} \tilde{T}_{\tilde{y}^{*}}^{*}=h_{f}\left(\tilde{T}_{f}-\tilde{T}\right), \quad-D \tilde{C}_{\tilde{y}^{*}}^{*}=k_{m}\left(\tilde{C}_{f}-\tilde{C}\right) \quad \text { at } \quad \tilde{y}^{*}=0, \\
& \tilde{u}^{*} \rightarrow 0, \quad \tilde{T}^{*} \rightarrow \tilde{T}_{\infty}, \quad \tilde{C}^{*} \rightarrow \tilde{C}_{\infty} \quad \text { at } \quad \tilde{y}^{*} \rightarrow \infty .
\end{aligned}
$$

Upon using Eqs (2.5) and (2.6), Eqs (2.2), (2.3) and (2.4) will take the following form 


$$
\begin{aligned}
& -v_{0} \tilde{u}_{\tilde{y}^{*}}^{*}=v \tilde{u}_{\tilde{y}^{*} \tilde{y}^{*}}^{*}+g \beta_{l}\left(\tilde{T}^{*}-\tilde{T}_{\infty}\right)+g \beta^{*}\left(\tilde{C}^{*}-\tilde{C}_{\infty}\right)-\frac{v \tilde{u}^{*}}{K^{*}}-\frac{\sigma B_{0}^{2} \tilde{u}^{*}}{\rho}, \\
& -v_{0} \tilde{T}_{\tilde{y}^{*}}^{*}=\frac{\kappa}{\rho C_{p}} \tilde{T}_{\tilde{y}^{*} \tilde{y}^{*}}^{*}+\frac{v}{C_{p}}\left(\tilde{u}_{\tilde{y}^{*}}^{*}\right)^{2}-\frac{1}{\rho C_{p}} 4\left(\tilde{T}^{*}-\tilde{T}_{\infty}\right) I^{\prime}-\frac{\sigma B_{0}^{2}}{\rho C_{p}} \tilde{u}^{* 2}, \\
& -v_{0} \tilde{C}_{\tilde{y}^{*}}^{*}=D \tilde{C}_{\tilde{y}^{*} \tilde{y}^{*}}^{*}-k c \tilde{C}^{*} .
\end{aligned}
$$

Consider the following non-dimensional parameters

$$
\begin{aligned}
& f(\eta)=\frac{\tilde{u}^{*}}{v_{0}}, \quad \eta=\frac{v_{0} \tilde{y}^{*}}{v}, \quad \operatorname{Pr}=\frac{v \rho C_{p}}{\kappa}, \quad F=\frac{4 v I^{\prime}}{\kappa v_{0}^{2}}, \\
& \alpha=\frac{v_{0}^{2} K^{*}}{v^{2}}, \quad \theta(\eta)=\frac{\tilde{T}^{*}-\tilde{T}}{\tilde{T}_{f}-\tilde{T}_{\infty}}, \quad \phi(\eta)=\frac{\tilde{C}^{*}-\tilde{C}}{\tilde{C}_{f}-\tilde{C}_{\infty}} .
\end{aligned}
$$

Using the dimensionless quantities given by Eqs (2.11), the Eqs (2.8)-(2.10) will reduce into

$$
\begin{aligned}
& f^{\prime \prime}+f^{\prime}-\left(\frac{1}{\alpha}+M\right) f^{\prime}=-\operatorname{Gr} \theta-\operatorname{Gm} \phi, \\
& \theta^{\prime \prime}+\operatorname{Pr} \theta^{\prime}-F \theta=-E \operatorname{Pr} f^{\prime 2}-\operatorname{Pr} E M f^{2}, \\
& \phi^{\prime \prime}+\operatorname{Sc} \phi^{\prime}=\operatorname{Sc} K_{r} \phi,
\end{aligned}
$$

here the prime denotes differentiation w.r. to $\eta$.

$$
\begin{aligned}
& \mathrm{Gr}=\frac{g \beta_{1}\left(\tilde{T}_{f}-\tilde{T}_{\infty}\right) v^{2}}{\lambda v_{0}^{3}}, \quad \mathrm{Gm}=\frac{g \beta^{*}\left(\tilde{C}_{f}-\tilde{C}_{\infty}\right) v}{D v_{0}^{3}}, \\
& \mathrm{Sc}=\frac{\nu}{D}, \quad K_{r}=\frac{k c v}{v_{0}^{2}}, \quad M=\frac{\sigma v B_{0}^{2}}{\rho v_{0}^{2}}, \quad E=\frac{v_{0}^{2}}{C_{p}\left(\tilde{T}_{f}-\tilde{T}_{\infty}\right)} .
\end{aligned}
$$

The corresponding boundary conditions are

$$
\begin{aligned}
& \eta=0, \quad f=0, \quad \theta^{\prime}(0)=-\mathrm{Bi}_{1}(1-\theta(0)), \quad \phi^{\prime}(0)=-\mathrm{Bi}_{2}\left(1-\phi^{\prime}(0)\right), \\
& \eta \rightarrow \infty, \quad f \rightarrow 0, \quad \theta \rightarrow 0, \quad \phi \rightarrow 0
\end{aligned}
$$

where $\mathrm{Bi}_{l}$ and $\mathrm{Bi}_{2}$ are Biot numbers and are given by

$$
\mathrm{Bi}_{l}=\frac{h_{f}}{k_{f}} \sqrt{\frac{v}{a}}, \quad \mathrm{Bi}_{2}=\frac{k_{f}}{D_{m}} \sqrt{\frac{v}{a}} .
$$




\section{Numerical method}

In the present investigation, a useful fifth-order Runge-Kutta Fehlberg scheme has been used to examine the flow. The influence of flow parameters on the dimensionless momentum, energy, and mass fields are examined. The $5^{\text {th }}$ order $\mathrm{R}-\mathrm{K}-\mathrm{Fehlberg}$ technique can be established as

$$
f=x_{1}, \quad f^{\prime}=x_{2}, \quad \theta=x_{3}, \quad \theta^{\prime}=x_{4}, \quad \phi=x_{5}, \quad \phi^{\prime}=x_{6} .
$$

In view of Eqs (3.1), Eqs (2.12)-(2.14) first-order ordinary differential equations as

$$
\begin{aligned}
& x_{1}^{\prime}=x_{2}, \\
& x_{2}^{\prime}=-x_{2}+\left(\frac{1}{\alpha}+M\right) x_{1}-\operatorname{Gr} x_{3}-\operatorname{Gm} x_{5}, \\
& x_{3}^{\prime}=x_{4}, \\
& x_{4}^{\prime}=-\operatorname{Pr} x_{4}+F x_{3}-E \operatorname{Pr} x_{2}^{2}-\operatorname{Pr} E M x_{1}^{2}, \\
& x_{5}^{\prime}=x_{6}, \\
& x_{6}^{\prime}=-\operatorname{Sc} x_{6}+\operatorname{Sc} K_{r} x_{5} .
\end{aligned}
$$

Respective boundary conditions are

$$
\begin{aligned}
& x_{1}(0)=0, \quad x_{2}(0)=m_{1}, \quad x_{3}(0)=m_{2}, \quad x_{4}(0)=-\mathrm{Bi}_{1}\left(1-x_{3}(0)\right), \\
& x_{5}(0)=m_{3}, \quad x_{6}(0)=-\mathrm{Bi}_{2}\left(1-x_{5}(0)\right) .
\end{aligned}
$$

here $m_{1}$ to $m_{3}$ are not known and they are resolute by the shooting method.

\section{Results and discussion}

The explanation of the problem is gained via the shooting technique. The various effects on motion fields are presented with the help of plotted graphs [2-13]. For fixed values as revealed in figures, the impact of theporous parameter on momentum description has been examined. The enhancing values of this parameter raise the velocity as shown in Fig.2. Figure 3 displayed to view the impact of Grashof number of the mass transfer on velocity and here too the trend is similar. The impact of the Grashof number of the heat transfer on velocity increases the corresponding profile and it is observed in Fig.4. It is because Grashof number is inversely proportional to viscous force and hence when values of both $\mathrm{Gr}$ and $\mathrm{Gm}$ increase the viscous force decreases which intern increases the flow velocity. The effect of Hartman number Mreduces the velocity description because of the Lorentz force which is a resistive force exerted against flow velocity and it can be seen in Fig.5.

The effect of variation in $\mathrm{Bi}_{l}$ is shown in Fig.6 on the temperature profile. The increasing values of $\mathrm{Bi}_{1}$ increase the temperature description. This is because as $\mathrm{Bi}_{l}$ enhances, heat transfer via convection takes place largely. Therefore, theheat profile increases with increasing $\mathrm{Bi}_{1}$. In Fig.7, the impact of $E$ on temperature has been presented. The enhance in $E$ increases the temperature description. When the Eckert number increases viscous dissipation of the fluid increases which intern increases the temperature of the fluid. Figures 8 and 9 are designed to analyze the effect of $F$ and $\mathrm{Gr}$ on temperature profiles respectively. 
The increasing values of $F$ reducing the temperature while enhancing the values of Gr enhances the temperature profile. In Fig.10, the impact of Pr on temperature can be observed. An enhance inPr reduces the temperature description because when thePrandtl number increases thermal diffusivity decreases. The effects of $\mathrm{Bi}_{2}, K_{r}$ and $\mathrm{Sc}$ on $\varphi(\eta)$ are shown in the Figs 11, 12 and 13. The increasing value of $\mathrm{Bi}_{2}$ increases the $\varphi(\eta)$ profile. This is because of the mass convection increases the concentration of the fluid. The uplift of $K_{r}$ and Sc decreases the $\varphi(\eta)$ description. In case of $K_{r}$, chemical reaction consumes the chemical in the liquid and therefore, the mass description decreases. Again, one can notice that as Sc raises, mass description declines. This is because of the truth that Scis conversely proportional to concentration diffusivity. Consequently, an enhance in values of $\mathrm{Sc}$ decreases the concentration diffusion and in turn decreases mass description.

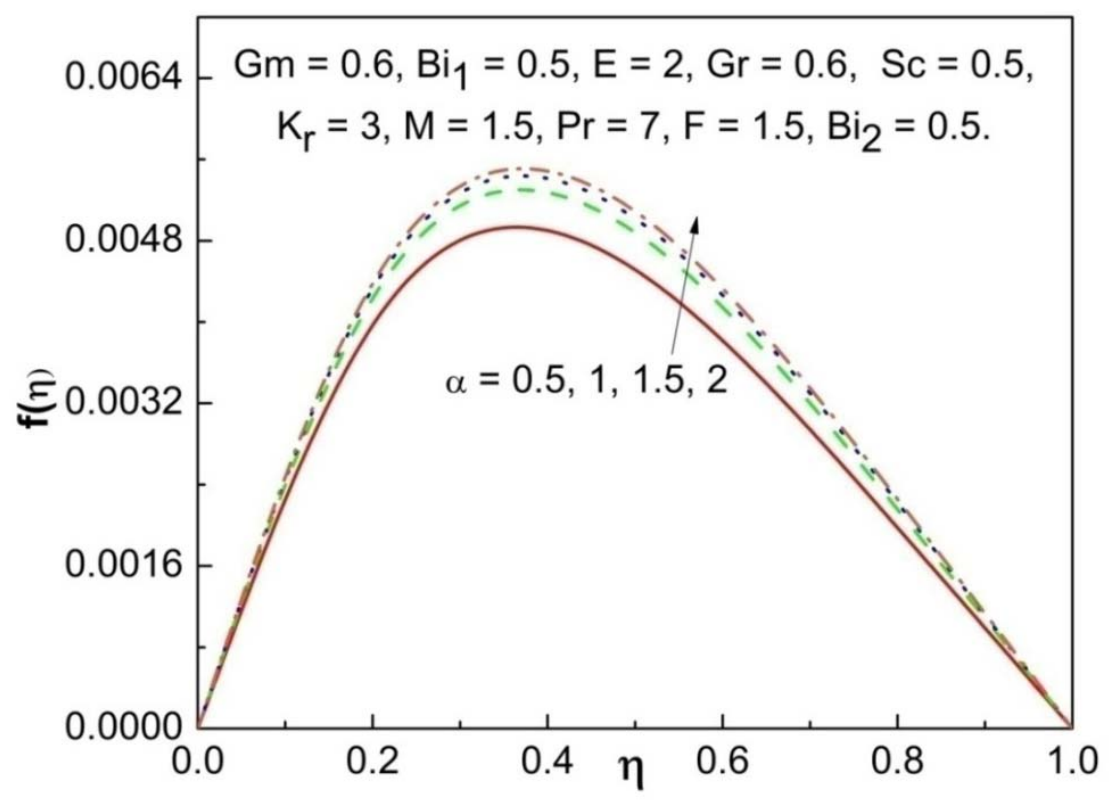

Fig.2. Consequence of $\alpha$ on $f(\eta)$.

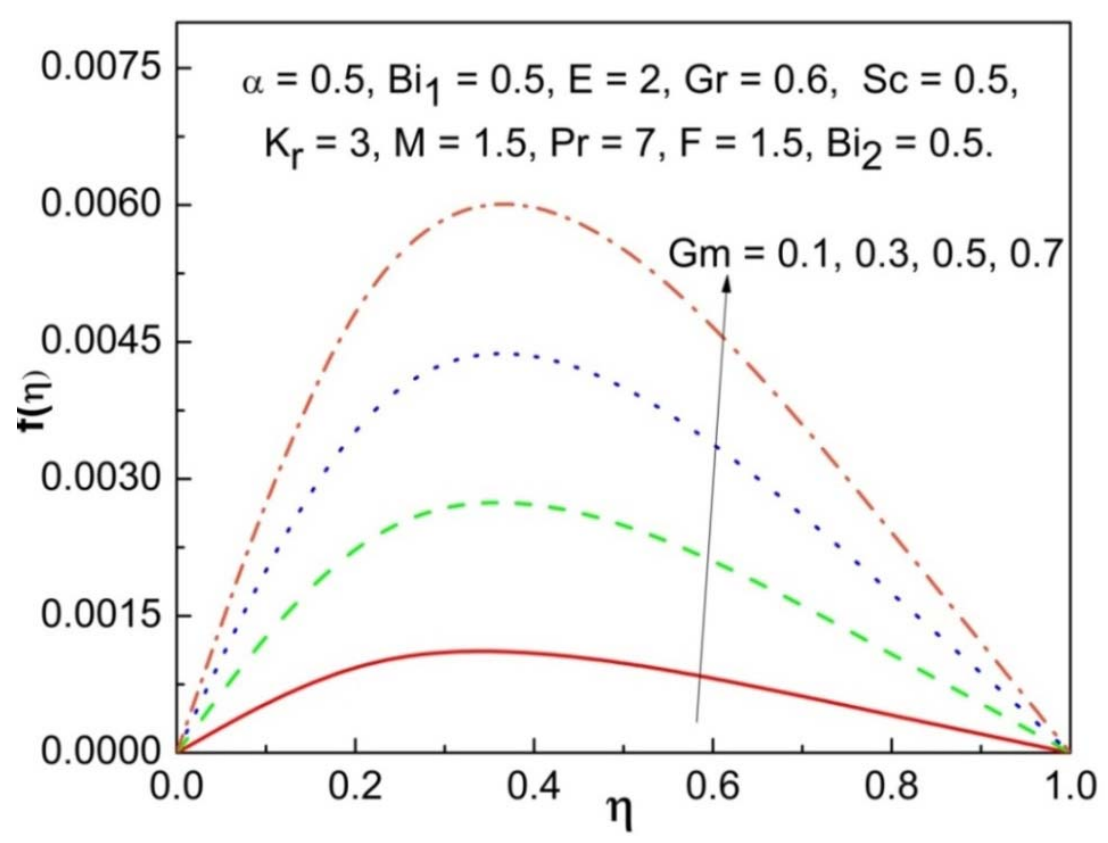

Fig.3. Consequence of Gm on $f(\eta)$. 


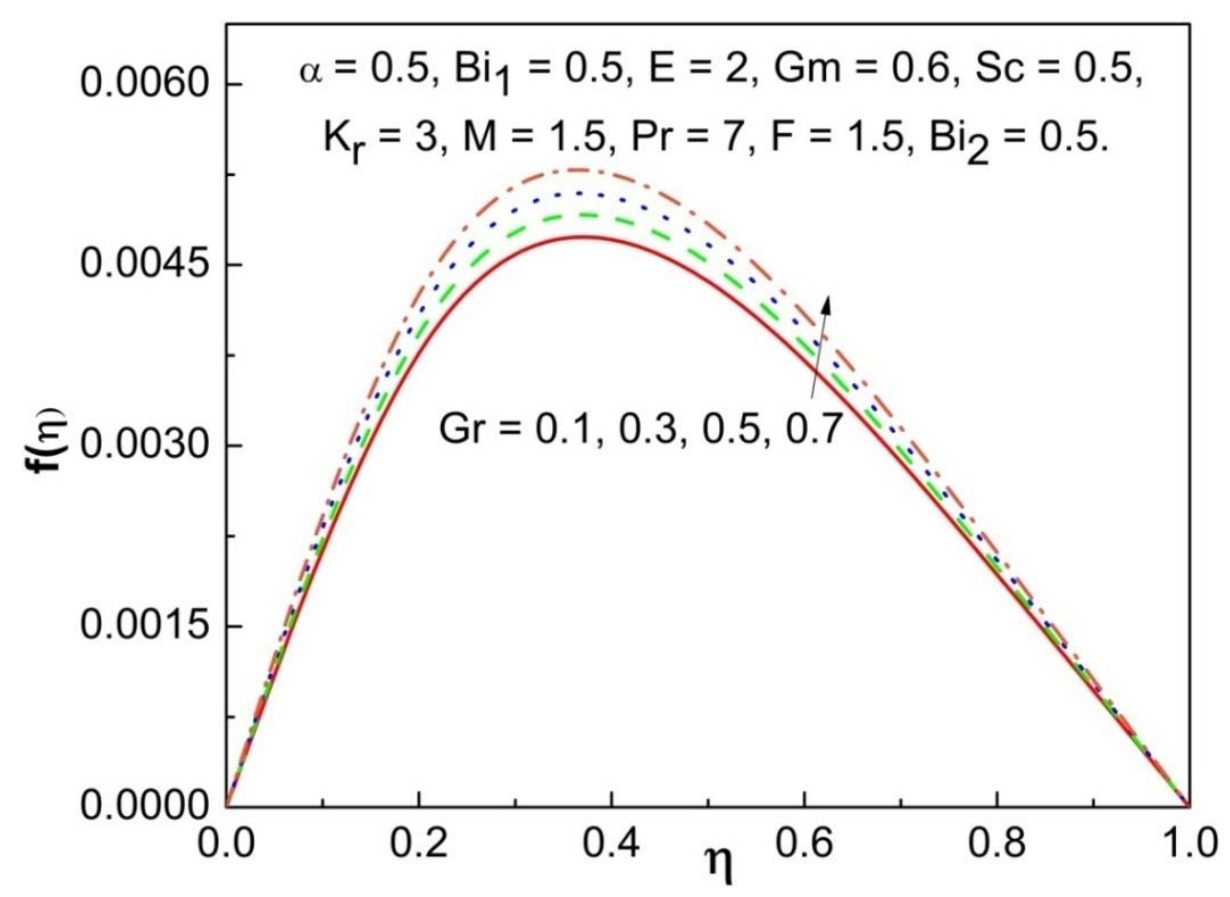

Fig.4. Consequence of Gr on $f(\eta)$.

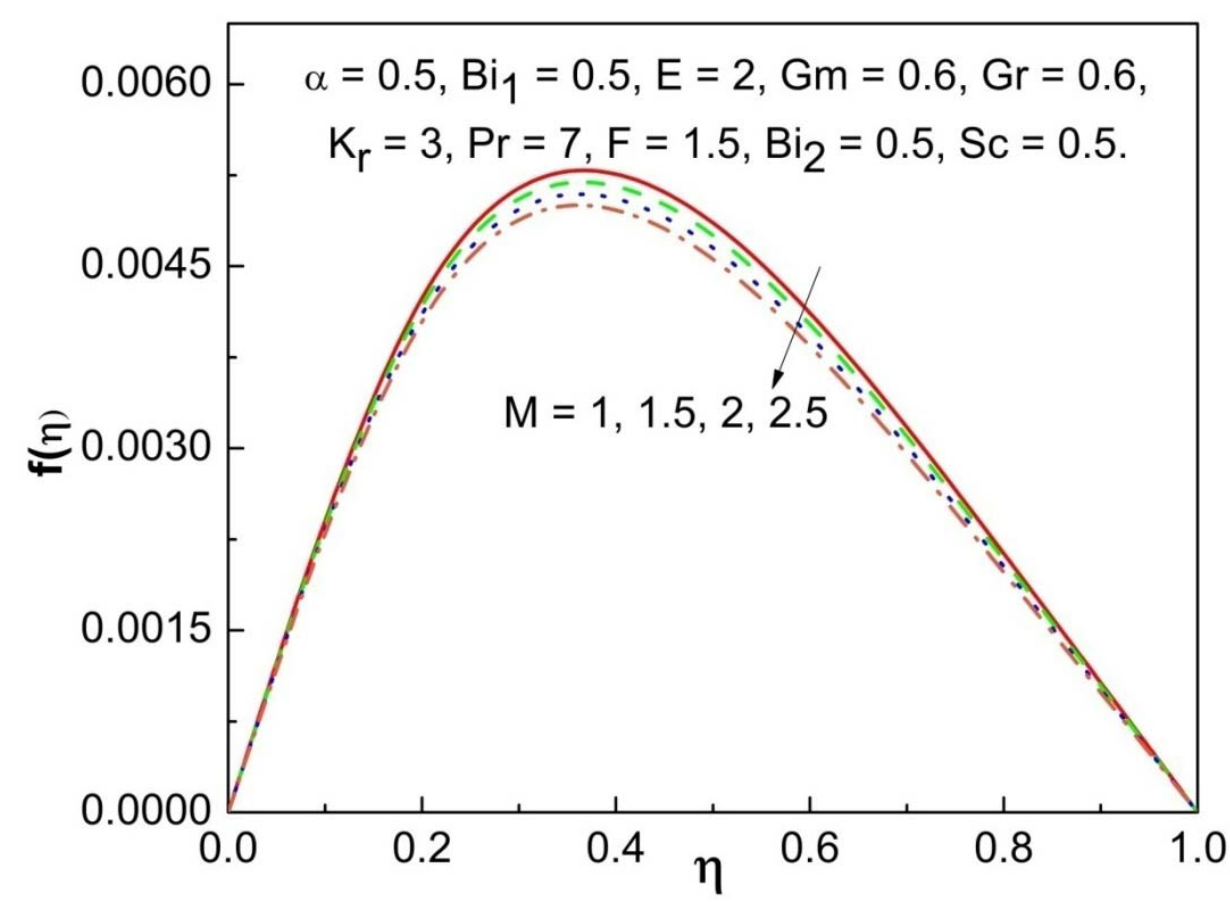

Fig.5. Consequence of $M$ on $f(\eta)$. 


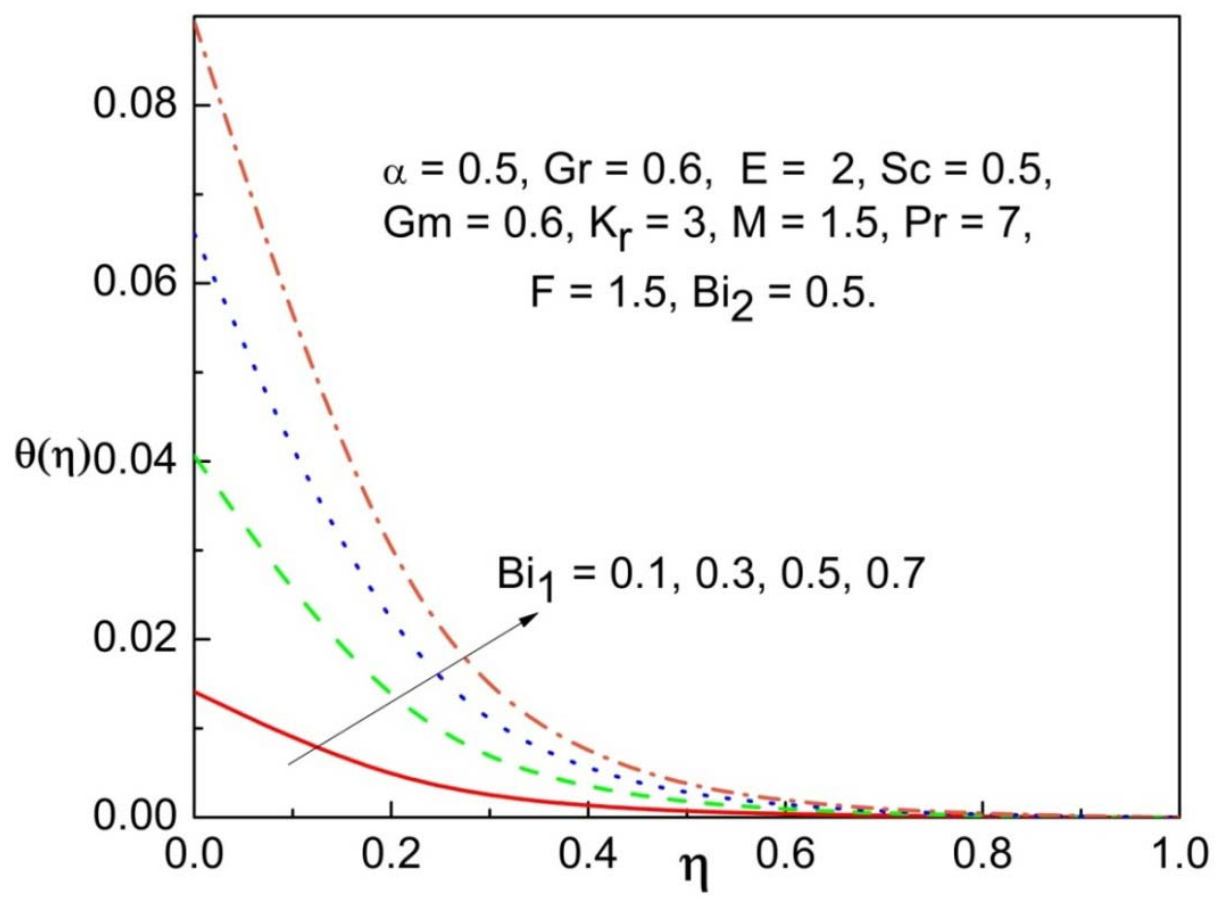

Fig.6. Consequence of $\mathrm{Bi}_{l}$ on $\theta(\eta)$.

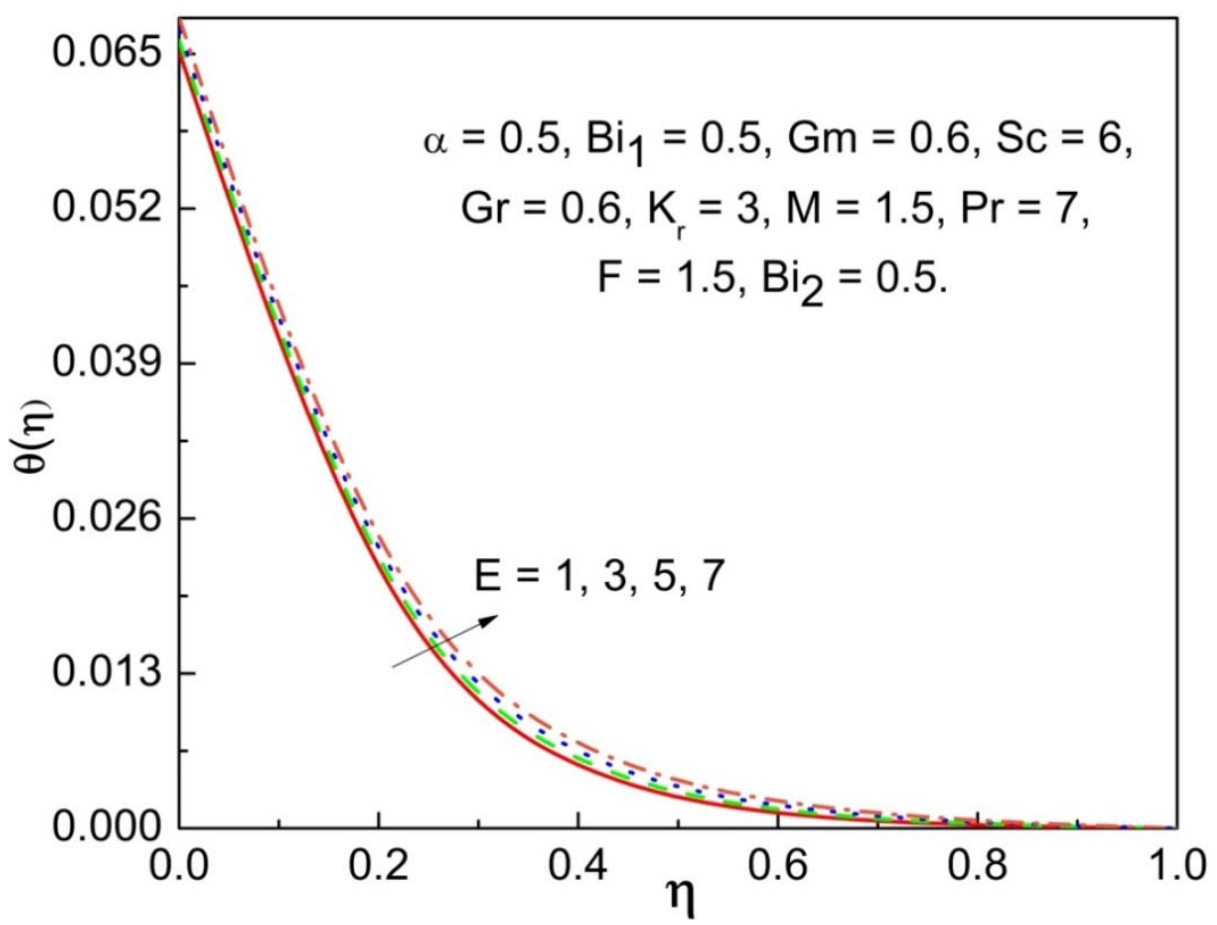

Fig.7. Consequence of $E$ on $\theta(\eta)$. 


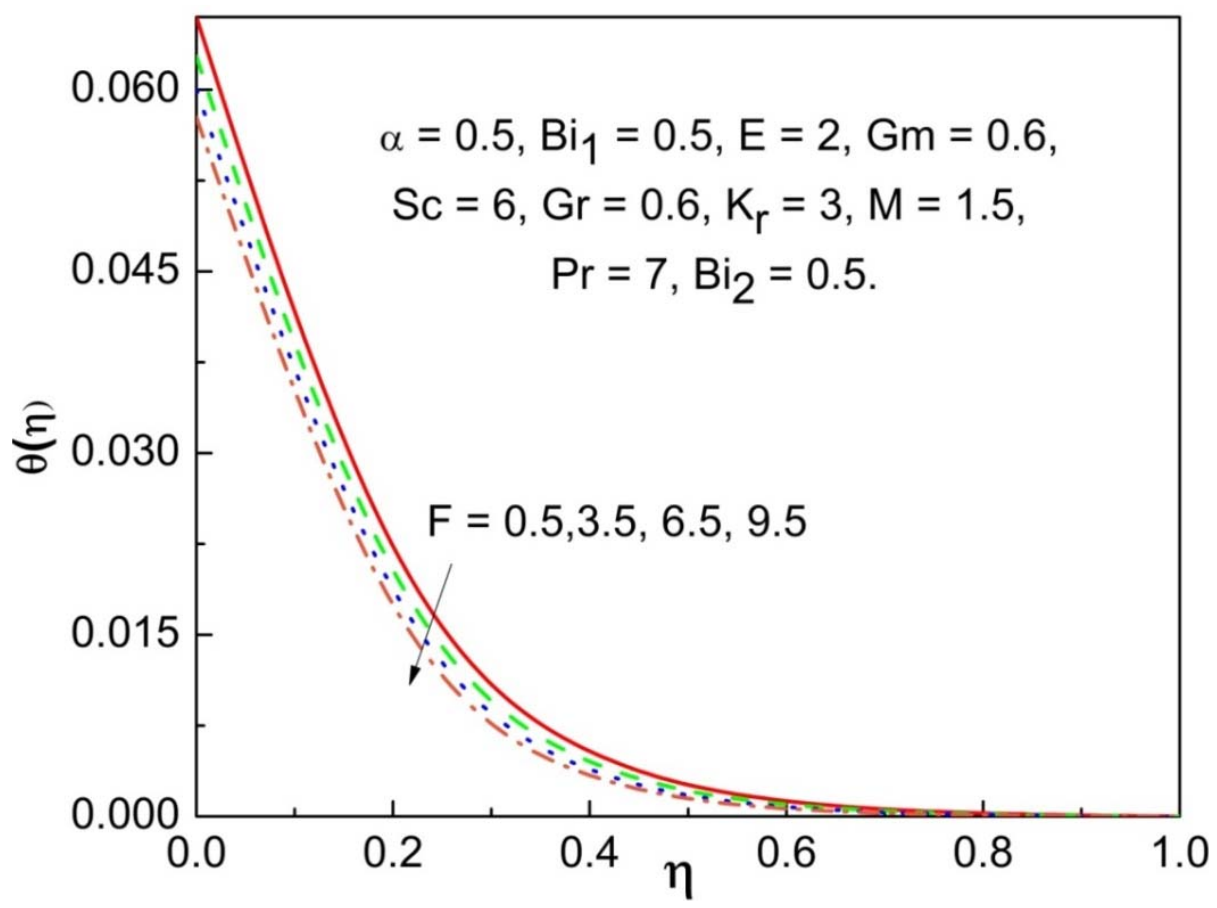

Fig.8. Consequence of $F$ on $\theta(\eta)$.

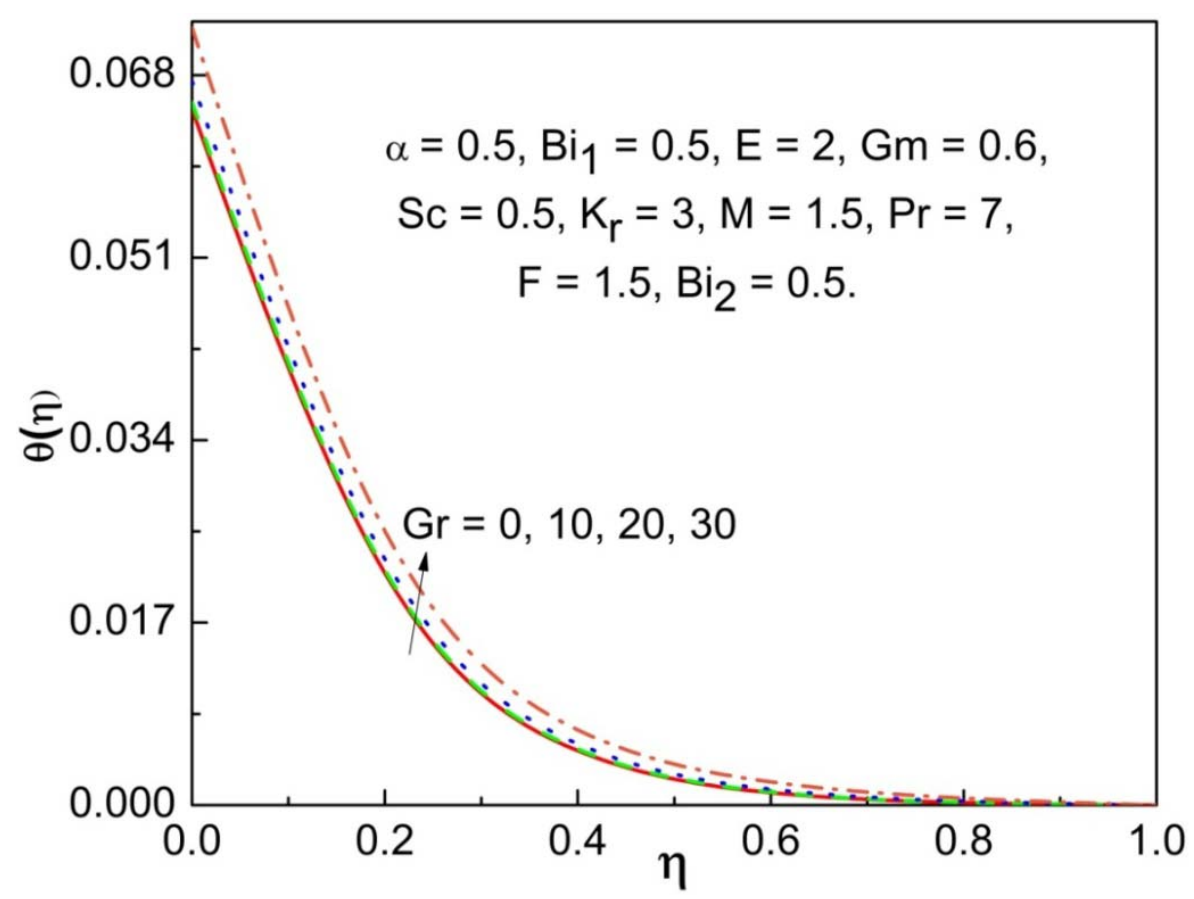

Fig.9. Consequence of Gr on $\theta(\eta)$. 


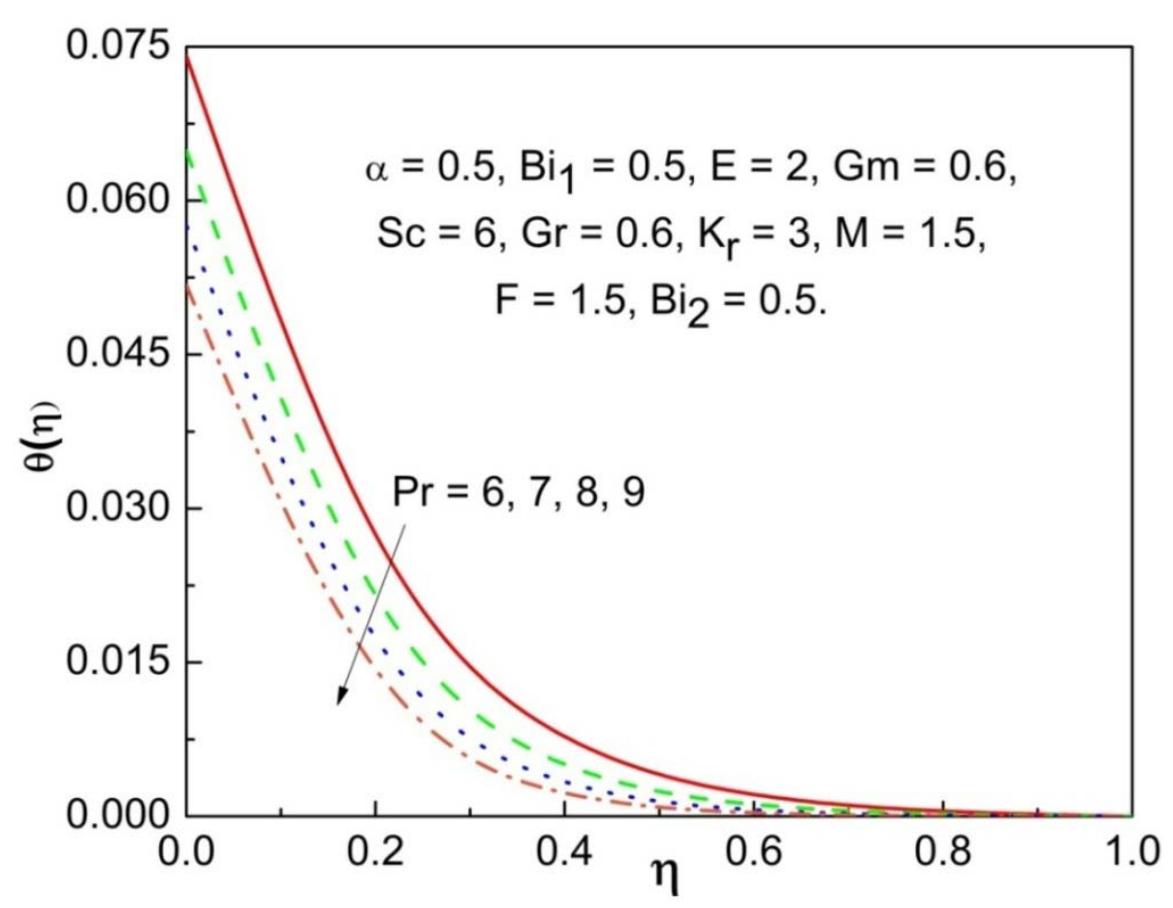

Fig.10. Consequence of $\operatorname{Pr}$ on $\theta(\eta)$.

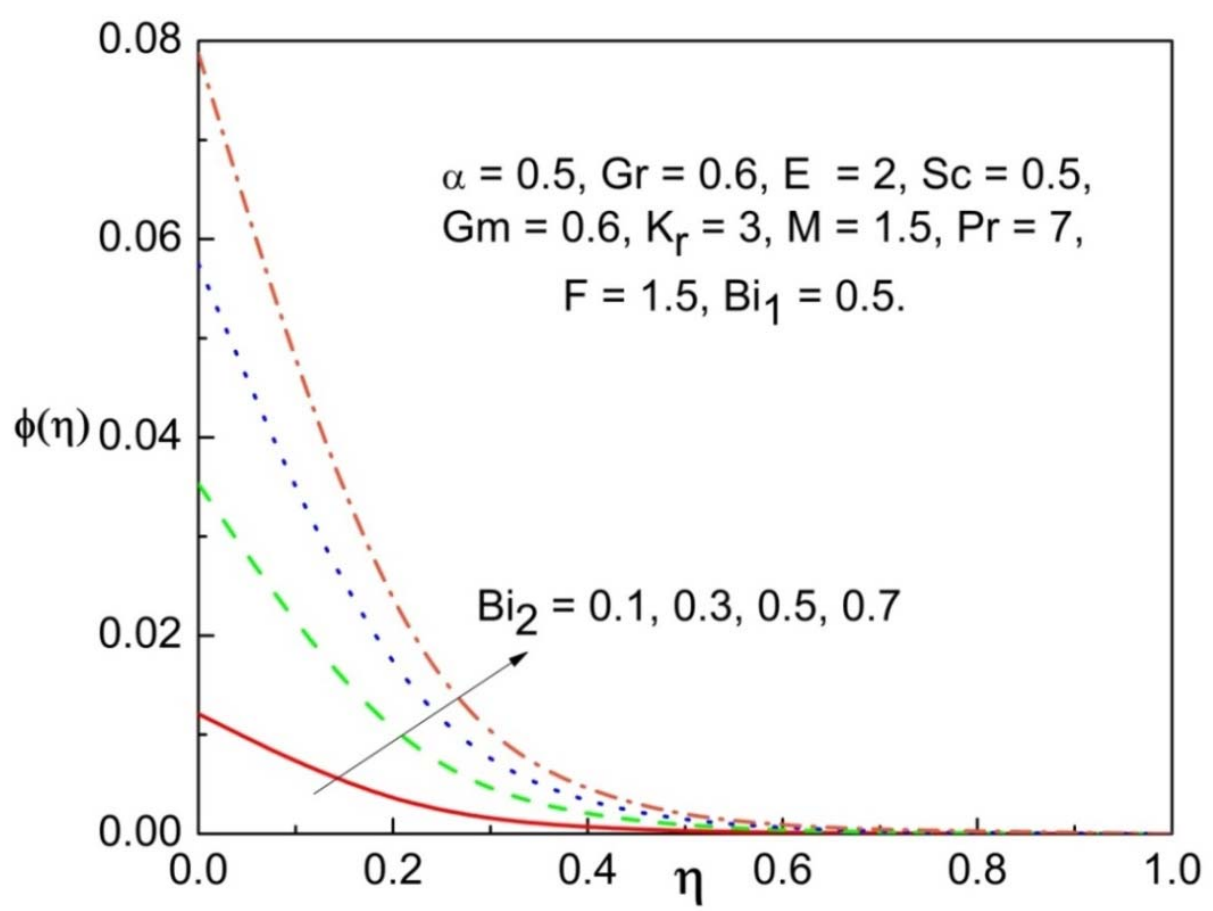

Fig.11. Consequence of $\mathrm{Bi}_{2}$ on $\phi(\eta)$. 


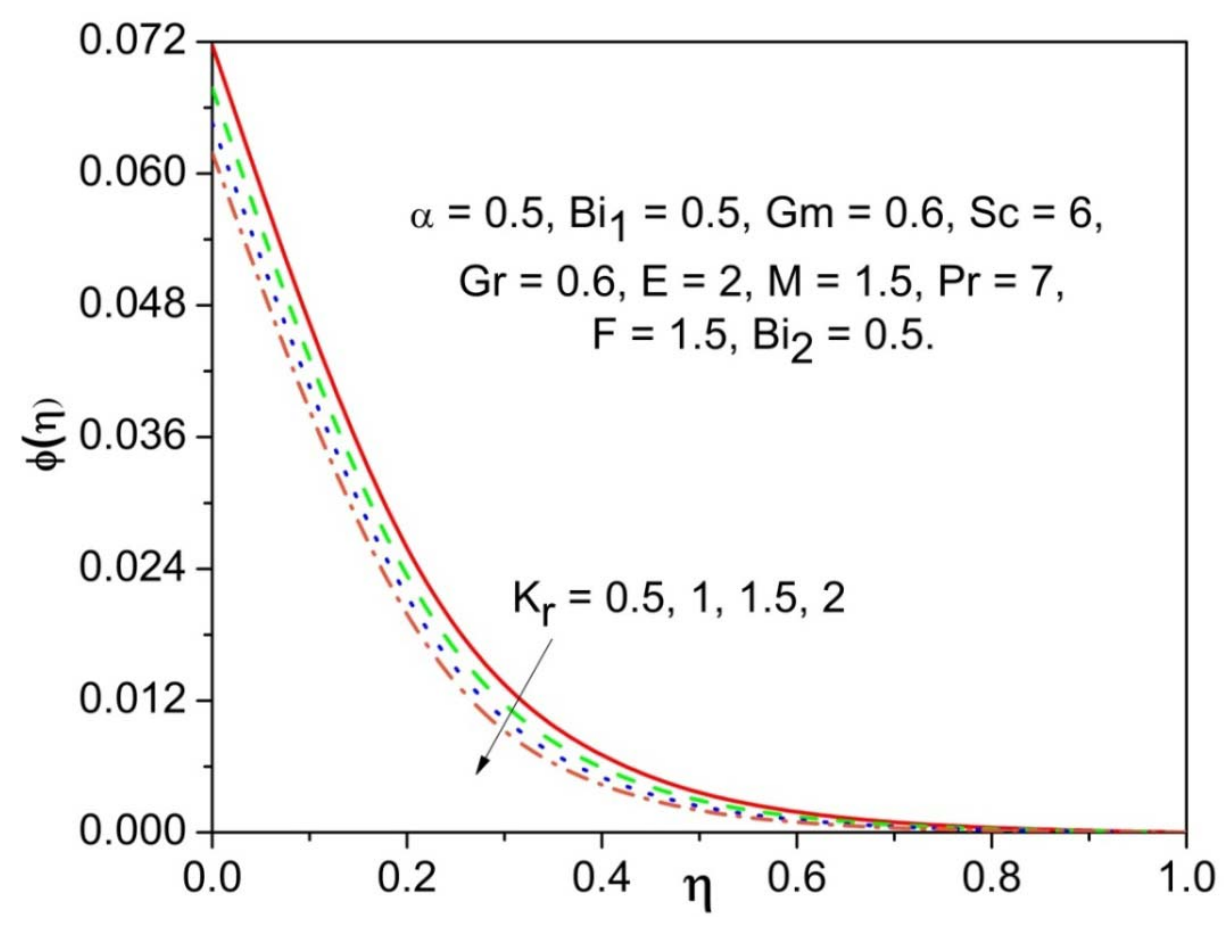

Fig.12. Consequence of $K r$ on $\phi(\eta)$.

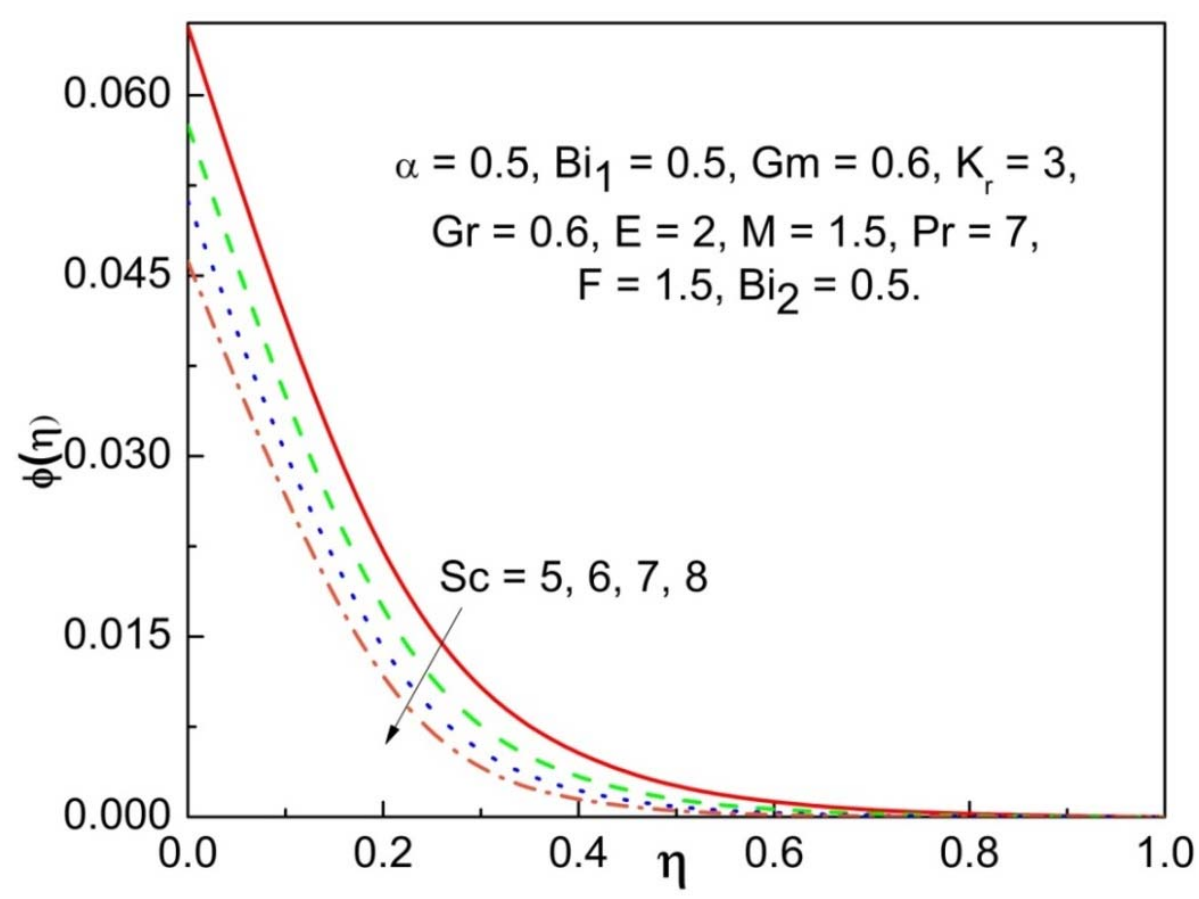

Fig.13. Consequence of Sc on $\phi(\eta)$. 


\section{Conclusion}

The effect of thermal radiation and Joule heating on magnetohydrodynamic boundary layer motion are explored by incorporating the convective heat and mass conditions. The major results of the study are summarized as follows;

- Impact of permeability parameter, Grashoff number of heat and mass transfer increases the velocity profile and the opposite trend can be observed for the magnetic parameter.

- Impact of Biot number of heat transfer, Eckert number, Grashoff number of heat transfer enhances the temperature whereas the radiation parameter and Prandtl number reduces the corresponding description.

- The impact of the Biot number of mass transfer enhances the concentration profile whereas the chemical reaction parameter and Schmidt number reduces the profile.

\section{Acknowledgments}

The authors are grateful to Prof. G.C. Sharma, Agra University, Agra, India for his help and valuable suggestions to prepare this article and thanks to reviewers also.

Conflict of Interest: The authors declare that they have no conflict of interest.

\section{Nomenclature}

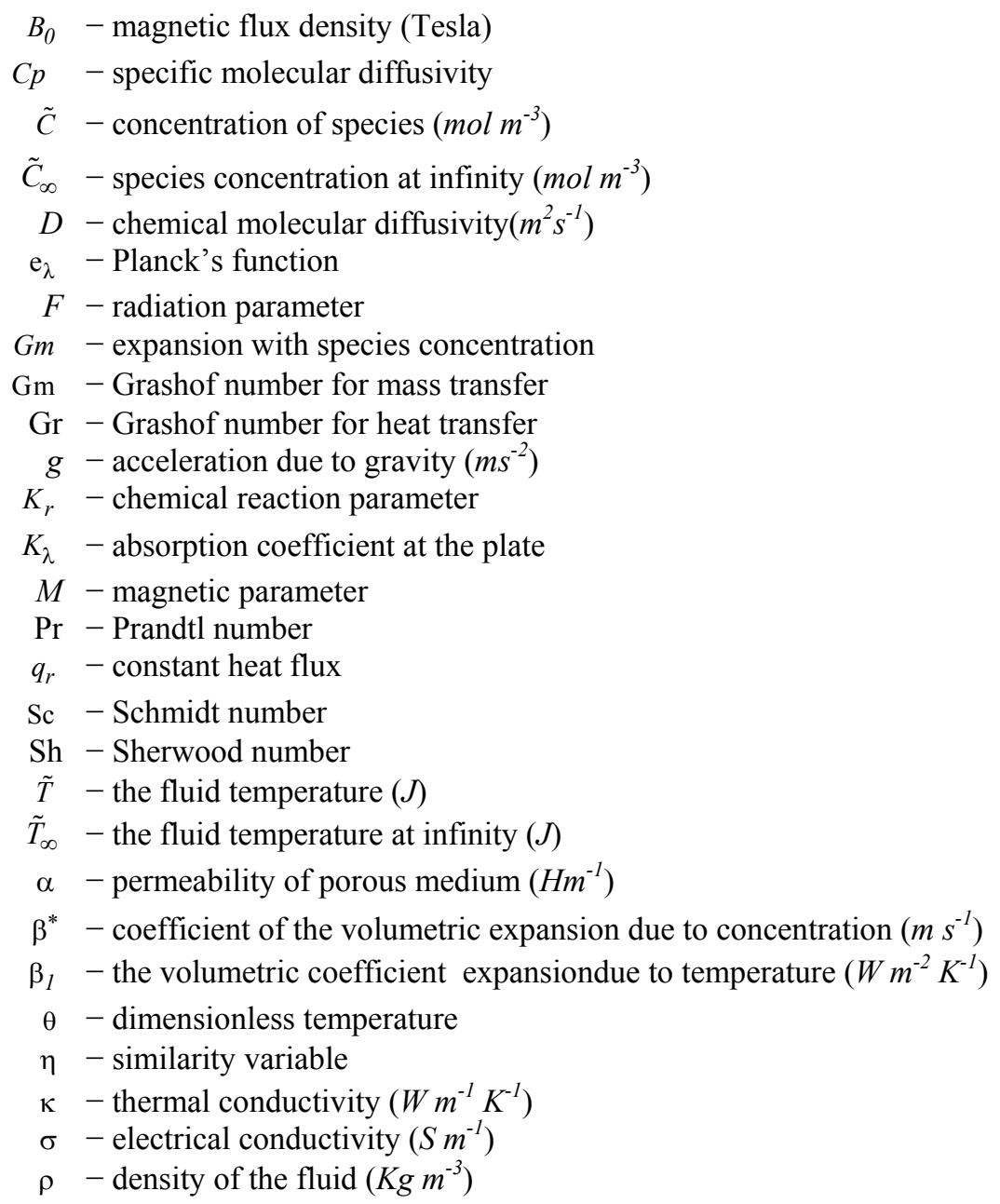


$v_{0}-$ scale of suction velocity

$v \quad$ - kinematic viscosity $\left(\mathrm{m}^{2} \mathrm{~s}^{-1}\right)$

$\mu \quad-$ dynamic viscosity $\left(K g m^{-1} s^{-1}\right)$

$v_{0}-$ constant suction velocitynormal to the plate

\section{References}

[1] Sivaraj R. and Rushi Kumar B. (2013): Chemically reacting dusty viscoelastic fluid flow in an irregular channel with convective boundary.- Ain Shams Engineering Journal, vol.4, No.1, pp.93-101.

[2] RamReddy C., Murthy P.V.S.N., Chamkha A.J. and Rashad A.M. (2013): Soret effect on mixed convection flow in a nanofluid under convective boundary condition.- International Journal of Heat and Mass Transfer, vol.64, pp.384392.

[3] Hayat T., Saeed Y., Asad S. and Alsaedi A. (2015): Soret and Dufour effects in the flow of Williamson fluid over an unsteady stretching surface with thermal radiation.- Zeitschrift für Naturforschung A, vol.70, No.4, pp.235-243.

[4] Uddin M.J, Rostami B., Rashid M.M. and Rostami P. (2016): Similarity and analytical solutions of free convective flow of dilatant nanofluid in a Darcian porous medium with multiple convective boundary conditions. - Alexandria Engineering Journal, vol.55, No.1, pp.263-274.

[5] Sheikholeslami M., Hayat T. and Alsaedi A. (2016): MHD free convection of Al2O3-water nanofluid considering thermal radiation: A numerical study.- International Journal of Heat and Mass Transfer, vol.96, No.5, pp.513-524.

[6] Sharma R.P., Ibrahim S.M., Jain M. and Mishra S.R. (2018): Chemical reaction effect on MHD rotating fluid over a vertical plate with variable thermal conductivity: A numerical study.- Indian Journal of Pure and Applied Physics, vol.56, pp.732-740

[7] Mishra S.R., Pattnaik P.K., Bhatti M.M. and Abbas T. (2017): Analysis of heat and mass transfer with MHD and chemical reaction effects on viscoelastic fluid over a stretching sheet.- Indian Journal of Physics, vol.91, pp.12191227.

[8] Gireesha B.J., Archana M., Prasannakumara B.C., Gorla R.S.R. and Makinde O.D. (2017): MHD three-dimensional double-diffusive flow of Casson nanofluid with buoyancy forces and nonlinear thermal radiation over a stretching surface.- International Journal of Numerical Methods for Heat and Fluid Flow, vol.27, No.12, pp.2858-2878.

[9] Ramzan M., Bilal M., Chung J.D. and Mann A.B. (2015): Flow of Casson nanofluid with viscous dissipation and convective conditions: a mathematical model.- Journal of Central South University, vol.22, No.3, pp.1132-1140.

[10] Hartmann J. and Dynamics I. (1937): Hg-dynamics I. Theory of the laminar flow of an electrically conductive liquid in a homogeneous magnetic field.- Math. Phys. Medd.: København, Levin and Munksgaard, Ejnar Munksgaard.

[11] Archana M., Gireesha B.J., Venkatesh P. and Reddy M.G. (2017): Influence of nonlinear thermal radiation and magnetic field on the three-dimensional flow of a Maxwell nanofluid.- Journal of Nanofluids, vol.6, No.2, pp.232242.

[12] Makinde O.D., Khan Z.H., Ahmad R. and Khan W.A. (2018): Numerical study of unsteady hydromagnetic radiating fluid flow past a slippery stretching sheet embedded in a porous medium. - Physics of Fluids, vol.30, No.8, pp.083601.

[13] Rashidi M.M., Rostami B., Freidooni Meher N. and Abbasbandy S. (2014): Free convective heat and mass transfer for MHD fluid flow over a permeable vertical stretching sheet in the presence of the radiation and buoyancy effects.- Ain Shams Engineering Journal, vol.5, No.3, pp.901-912.

[14] Ramzan M. and Bilal M. (2015): Time-dependent MHD nano-second grade fluid flow induced by permeable vertical sheet with mixed convection and thermal radiation. - PloS one, vol.10, No.5, pp.e0124929.

[15] Kataria H.R. and Patel H.R. (2016): Soret and heat generation effects on MHD Casson fluid flow past an oscillating vertical plate embedded through a porous medium.- Alexandria Engineering Journal, vol.55, No.3, pp.2125-2137. 
[16] Khan I., Malik M.Y., Hussain A. and Salahuddin T. (2017): Effect of homogenous-heterogeneous reactions on MHD Prandtl fluid flow over a stretching sheet. - Results in Physics, vol.7, pp.4226-4231.

[17] Sheikholeslami M., Ganji D.D., Javed M.Y. and Ellahi R. (2015): Effect of thermal radiation on magnetohydrodynamic nanofluid flow and heat transfer by means of two-phase model. - Journal of Magnetism and Magnetic Materials, vol.374, pp.36-43.

[18] Zeeshan A., Majeed A. and Ellahi R. (2016), Effect of magnetic dipole on viscous ferrofluid past a stretching surface with thermal radiation.- Journal of Molecular Liquids, vol.215, pp.549-554.

[19] Nayak M.K., Akbar N.S., Pandey V.S., Khan Z.H. and Tripathi D. (2017): 3D free convective MHD flow of nanofluid over a permeable linear stretching sheet with thermal radiation, Powder Technology. - Vol.315, pp.205-215.

[20] Dawar A., Shah Z., Idrees M., Khan W., Islam S. and Gul T. (2018): Impact of thermal radiation and heat source/sink on Eyring-Powell fluid flow over an unsteady oscillatory porous stretching surface. - Mathematical and Computational Applications, vol.23, No.2, pp.1-18.

[21] Nawaz M., Zeeshan A., Ellahi R., Abbasbandy S. and Rashidi S. (2015): Joules and Newtonian heating effects on stagnation point flow over a stretching surface by means of genetic algorithm and Nelder-Mead method. International Journal of Numerical Methods for Heat and Fluid Flow, vol.25, No.3, pp.665-684.

[22] Hayat T., Shafique M., Tanveer A. and Alsaedi A. (2016): Magnetohydrodynamic effects on peristaltic flow of hyperbolic tangent nanofluid with slip conditions and Joule heating in an inclined channel. - International Journal of Heat and Mass Transfer, vol.102, pp.54-63.

[23] Dogonchi A.S. and Ganji D.D. (2017): Analytical solution and heat transfer of two-phase nanofluid flow between non-parallel walls considering Joule heating effect. - Powder Technology, vol.318, pp.390-400.

[24] Muhammad T., Hayat T., Shehzad S.A. and Alsaedi A. (2018): Viscous dissipation and Joule heating effects in MHD 3D flow with heat and mass fluxes.- Results in Physics, vol.8, pp.365-371.

[25] CogleyA.C., VincentiW.G. and GillS.E. (1968): Differential approximation for radiative transfer in a non-gray gas near equilibrium.- AIAA J., vol.6, pp.551-553.

[26] Kumar G., Ramesh G.K., Shehzad S.A. and Gireesha B.J. (2020): Magneto Prandtl nanofluid past a stretching surface with non-linear radiation and chemical reaction. - Journal of Computational and Applied Research in Mechanical Engineering (JCARME), vol.9, No.2, pp.275-285.

Received: February 2, 2019

Revised: March 25, 2020 